\title{
Vector-Valued Entire Functions of Bounded Index Satisfying a Differential Equation*
}

\author{
L. F. Heath**
}

(September 27, 1977)

\begin{abstract}
The concept of complex valued entire functions of bounded index is extended to $C^{n}$-valued entire functions by replacing the absolute value in the definition of an entire function of bounded index by the maximum of the absolute values of the components. If the components of a $C^{n}$-valued entire function are of bounded index, then the function is also of bounded index; however a $C^{n}$-valued function may be of bounded index without all of its components being of bounded index. Solutions of certain linear differential equations are related to $C^{n}$-valued functions of bounded index.
\end{abstract}

Key words: Bounded index; $C^{n}$-valued functions; entire functions; linear differential equations.

\section{Introduction}

If $f$ is a complex-valued entire function, then $f$ is said to be of bounded index of index $N$ if $N$ is the least non-negative integer such that

$$
\frac{\left|f^{(j)}(z)\right|}{j !} \leq \max _{0 \leq i \leq N} \frac{\left|f^{(i)}(z)\right|}{i !}
$$

for all integers $j \geq 0$ and all $z \in C\left([1],{ }^{1}[2]\right)$.

In this paper, we are concerned with one possible extension of this concept to vector-valued functions.

If $f_{i}, i=1,2, \ldots, n$, are complex-valued entire functions, then $F(z)=\left[f_{1}(z) f_{2}(z) \cdots f_{n}(z)\right]$ is a $C^{n}$ valued entire function (we write $C^{n}$-valued functions in this manner for convenience throughout this paper regardless of whether they are to be interpreted as row vectors or column vectors) and we write $\|F(z)\|=$ $\max \left\{\left|f_{i}(z)\right| \mid i=1,2, \ldots, n\right\}$ and $F^{\prime}(z)=\left[f_{1}^{\prime}(z) f_{2}^{\prime}(z) \ldots f_{n}^{\prime}(z)\right]$.

Definition: A $C^{n}$-valued entire function $F$ is said to be of bounded index of index $N$ if $N$ is the least non-negative integer such that

$$
\frac{\left\|F^{(j)}(z)\right\|}{j !} \leq \max _{0 \leq i \leq N} \frac{\left\|F^{(i)}(z)\right\|}{i !}
$$

for all integers $j \geq 0$ and all $z \epsilon C$.

\section{Properties and Examples}

We first relate the index of a $C^{n}$-valued (vector-valued) entire function of bounded index to the index of each of its components (scalars). We use lower case letters to denote scalar functions and upper case letters to denote vector-valued functions.

Lemma 2.1: If $f_{k}$ is an entire function of bounded index of index $N_{k}$ for $k=1,2, \ldots, n$, then $F=$ $\left[f_{1} f_{2} \cdots f_{n}\right]$ is of bounded index of index $N \leq M=\max \left\{N_{k} \mid k=1,2, \ldots, n\right\}$.

AMS Subject Classification: 30A64; 34A20 (1970).

** Present address: University of Texas at Arlington, Arlington, Texas 76019

${ }^{1}$ Figures in brackets indicate the literature references at the end of this paper. 
Proof: For each integer $j \geq 0$ and each $k=1,2, \ldots, n$

$$
\frac{\left|f_{k}^{(j)}(z)\right|}{j !} \leq \max _{0 \leq i \leq N_{k}} \frac{\left|f_{k}^{(i)}(z)\right|}{i !}=\max _{0 \leq i \leq M} \frac{\left|f_{k}^{(i)}(z)\right|}{i !} \leq \max _{0 \leq i \leq M} \frac{\mid F^{(i)}(z) \|}{i !}
$$

Therefore, (1.1) is satisfied.

REMARK 1: Both equality and inequality are attainable in Lemma 2.1.

EXAMPLE 1: Let $f_{1}(z)=e^{2 z}, f_{2}(z)=e^{z}$, and $F=\left[f_{1} f_{2}\right]$. Then $N_{1}=1, N_{2}=0$, and $N=1$.

ExAmple 2: Let $f_{1}(z)=2, f_{2}(z)=z$, and $F=\left[f_{1} f_{2}\right]$. Then $N_{1}=0, N_{2}=1$, and $N=0$.

REMARK 2: It is possible for $N<\min \left\{N_{k} \mid k=1,2, \ldots, n\right\}$ in Lemma 2.1.

Example 3: Let $f_{1}(z)=z^{2}, f_{2}(z)=z-8$, and $F=\left[f_{1} f_{2}\right]$. Then $N_{1}=2, N_{2}=1$, and $N=0$.

REMARK 3: If $F$ is of bounded index, then its components may not be of bounded index.

Example 4: Let $f$ be of unbounded index such that $f-c$, where $c$ is any non-zero constant, is of bounded index [7, Theorem 2, p. 128]. Let $f_{1}=f$ and $f_{2}=f-c$. Let $N_{2}$ be the index of $f_{2}$ and let $F=\left[f_{1} f_{2}\right]$. Then $F$ is of bounded index of index $N \leq N_{2}$.

Proof: For any integer $j \geq 1$ and $z \epsilon C, f_{1}^{(j)}(z)=f_{2}^{(j)}(z)$ and so

$$
\frac{\left\|F^{(j)}(z)\right\|}{j !}=\max \left\{\frac{\left|f_{1}^{(j)}(z)\right|}{j !}, \frac{\left|f_{2}^{(j)}(z)\right|}{j !}\right\}=\frac{\left|f_{2}^{(j)}(z)\right|}{j !} \leq \max _{0 \leq i \leq N_{2}} \frac{\left|f_{2}^{(i)}(z)\right|}{i !} \leq \max _{0 \leq i \leq N_{2}} \frac{\left\|F^{(i)}(z)\right\|}{i !} .
$$

Therefore (1.1) holds for all integers $j \geq 0$ and all $z \in C$.

\section{Differential Equations With Constant Coefficients}

If $A=\left[a_{i j}\right]$ is an $n \times n$ matrix, we use the norm $\|A\|=\max _{1 \leq i \leq n} \sum_{j=1}^{n}\left|a_{i j}\right|$. If $F$ is a solution of the vector equation $F^{\prime}=A F+Q$, where $A$ is a matrix whose entries are entire functions and $Q$ is a column vector whose entries are entire functions, then $F$ is an entire function [4]. We will show that if $A$ is a constant matrix, then any solution of $F^{\prime}=A F$ is of bounded index and we will obtain an upper bound on its index.

Theorem 3.1: If $F$ is a solution of $F^{\prime}=A F$ where $A$ is a constant matrix, then $F$ is an entire function of bounded index of index $N \leq M$ where

$$
M=\min \{m \epsilon Z \mid m \geq 0 \text { and }\|A\| \leq m+1\} .
$$

Proof: Let $m$ be a non-negative integer such that $\|A\| \leq m+1$. Then $F^{(m+p)}(z)=A F^{(m+p-1)}(z)$ for all integers $p \geq 1$ and all $z \epsilon C$. Therefore

$$
\frac{\left\|F^{(m+p)}(z)\right\|}{(m+p) !} \leq \frac{\|A\|}{m+p} \frac{\left\|F^{(m+p-1)}(z)\right\|}{(m+p-1) !} \leq \frac{\left\|F^{(m+p-1)}(z)\right\|}{(m+p-1) !}
$$

and so

$$
\frac{\left\|F^{(j)}(z)\right\|}{j !} \leq \max _{0 \leq i \leq m} \frac{\left\|F^{(i)}(z)\right\|}{i !}
$$

for all integers $j \geq 0$ and all $z \epsilon C$.

Remark: Both equality and inequality are attainable in Theorem 3.1.

Example 5: Let $F(z)=\left[e^{2 z} e^{z}\right]$ and $A=\left[\begin{array}{ll}2 & 0 \\ 0 & 1\end{array}\right]$. Then $\|A\|=2$ and $M=1$ and $N=1$ (see example 1). 
Example 6: Let $F(z)=[1 / 8 z]$ and $A=\left[\begin{array}{ll}0 & 0 \\ 8 & 0\end{array}\right]$. Then $\|A\|=8$ and $M=7$; however $N=1$.

Theorem 3.1 does not tell us whether or not the components of $F$ are of bounded index; however every component of $F$ satisfies a linear differential equation of the form

$$
\alpha_{0} y^{(n)}+\alpha_{1 y}{ }^{(n-1)}+\ldots+\alpha_{n-1} g^{\prime}+\alpha_{n} \gamma=0
$$

where $\alpha_{k} \epsilon C, k=0,1,2, \ldots, n[3]$. Therefore we have

THEorem 3.2: If $F$ is a solution of $F^{\prime}=A F$ where $A$ is a constant matrix, then each component of $F$ is of bounded index.

Proof: Since each component of $F$ satisfies a differential equation of the form (3.2), each component is then of bounded index, by [5].

By the same type of argument as in Theorem 3.1 and Theorem 3.2, we have

Theorem 3.3: If $F$ is a solution of $F^{\prime}=A F+Q$ where $A$ is a constant matrix and $Q=\left[q_{i}\right]$ is a column vector whose entries are polynomials, then each component of $F$ is of bounded index and $F$ is of bounded index of index $N \leq \min \left\{m \epsilon Z^{+} \mid\|A\| \leq m+1\right.$ and $\left.m-1 \geq \max _{1 \leq i \leq n} \operatorname{deg} q_{i}\right\}$.

Proof: Let $m$ be a non-negative integer such that $\|A\| \leq m+1$ and $m-1 \geq \max _{1 \leq i \leq n} \operatorname{deg} q_{i}$. Then for any integer $p \geq 1$, we have $F^{(m+p)}=A F^{(m+p-1)}+Q^{(m+p-1)}=A F^{(m+p-1)}$ and

$$
\frac{\left\|F^{(m+p)}(z)\right\|}{(m+p) !} \leq \frac{\|A\|}{m+p} \frac{\left\|F^{(m+p-1)}(z)\right\|}{(m+p-1) !} \leq \frac{\left\|F^{(m+p-1)}(z)\right\|}{(m+p-1) !} .
$$

Therefore, $\frac{\left\|F^{(m+p)}(z)\right\|}{(m+p) !} \leq \frac{\left\|F^{(m)}(z)\right\|}{m !}$ for all $p \geq 1$ and all $z \epsilon C$. Hence (1.1) holds for all $j \geq 0$ and all $z \epsilon C$.

\section{Differential Equations With Rational Coefficients}

In [6], it is shown that if $f$ is an entire solution of (3.2) where $\alpha_{i}$ are polynomials and $\operatorname{deg} \alpha_{i} \leq \operatorname{deg} \alpha_{0}$ for $i=1,2, \ldots, n$, then $f$ is of bounded index. We will show a comparable result for vector equations.

Theorem 4.1: If $F$ is an entire solution of $F^{\prime}=A F+Q$ where $A=\left[r_{i j}\right]$ is a matrix whose entries are rational functions which are bounded at infinity and $Q$ is a vector whose entires are rational functions which are bounded at infinity, then $F$ is a function of bounded index.

Proof: Let $p_{0}$ be the least common denominator of the $r_{i j}$ s and $q_{i}$ s where $Q=\left[q_{i}\right]$. Let $b_{i j}=p_{0} r_{i j}$ and $p_{i}=p_{o} q_{i}$. Then $b_{i j}$ and $p_{i}$ are polynomials of degree $\leq l=\operatorname{deg} p_{0}$. Let $B=\left[b_{i j}\right]$ and $P=\left[p_{i}\right]$ and so $F$ is a solution of

$$
p_{0} F^{\prime}=B F+P \text {. }
$$

Differentiating $N$-times where $N \geq l+1$, we obtain

$$
\sum_{k=0}^{N}\left(\begin{array}{l}
N \\
k
\end{array}\right) p_{0}{ }^{(k)} F^{(N+1-k)}=\sum_{k=0}^{N}\left(\begin{array}{l}
N \\
k
\end{array}\right) B^{(k)} F^{(N-k)}
$$

which simplifies into

$$
p_{0} F^{(N+1)}=\sum_{k=0}^{l}\left(\begin{array}{l}
N \\
k
\end{array}\right) B^{(k)} F^{(N-k)}-\sum_{k=1}^{l}\left(\begin{array}{l}
N \\
k
\end{array}\right) p_{0}{ }^{(k)} F^{(N+1-k)}
$$

since $\operatorname{deg} b_{i j} \leq l=\operatorname{deg} p_{0}$. 
Therefore

$$
\frac{F^{(N+1)}}{(N+1) !}=\frac{1}{N+1} \sum_{k=0}^{l} \frac{1}{k !} \frac{B^{(k)}}{p_{0}} \frac{F^{(N=k)}}{(N-k) !}-\sum_{k=1}^{l} \frac{1}{k !}\left(1-\frac{k}{N+1}\right) \frac{p_{0}^{(k)}}{p_{0}} \frac{F^{(N+1-k)}}{(N+1-k) !}
$$

Since $\frac{p_{0}{ }^{(k)}(z)}{p_{0}(z)} \rightarrow 0$ as $|z| \rightarrow \infty$ for $k=1,2, \ldots, l$, there exists $T>0$ such that

$$
\sum_{k=1}^{l} \frac{1}{k !}\left|\frac{p_{0}{ }^{(k)}(z)}{p_{0}(z)}\right|<\frac{1}{2} \text { if }|z| \geq T
$$

Since $\operatorname{deg} b_{i j} \leq l, \sum_{k=0}^{l} \frac{1}{k !} \frac{\left\|B^{(k)}(z)\right\|}{\left|p_{0}(z)\right|} \leq H$, a constant, if $|z| \geq T$. Choose $N_{1} \geq l+1$ so that $\frac{1}{N_{1}+1} H<\frac{1}{2}$.

Then

$$
\frac{1}{N+1} \sum_{k=0}^{l} \frac{1}{k !} \frac{\left\|B^{(k)}(z)\right\|}{\left|p_{0}(z)\right|}<\frac{1}{2} \text { if } N \geq N_{1} \text { and }|z| \geq T
$$

Combining (4.2), (4.3), and (4.4) if $N \geq N_{1}$ and $|z| \geq T$, we have

$$
\begin{aligned}
\frac{\left\|F^{(N+1)}\right\|}{(N+1) !} & \leq\left[\frac{1}{N+1} \sum_{k=0}^{l} \frac{1}{k !} \frac{\left\|B^{(k)}\right\|}{p_{0}}+\sum_{k=1}^{l} \frac{1}{k !}\left|\frac{p_{0}{ }^{(k)}}{p_{0}}\right|\right] \times \max _{0 \leq i \leq N} \frac{\left\|F^{(i)}\right\|}{i !} \\
& \leq \max _{0 \leq i \leq N} \frac{\left\|F^{(i)}\right\|}{i !} .
\end{aligned}
$$

Therefore $\frac{\left\|F^{(N+1)}(z)\right\|}{(N+1) !} \leq \max _{0 \leq i \leq N_{1}} \frac{\left\|F^{(i)}(z)\right\|}{i !}$ for all $N \geq N_{1}$ and $|z| \geq T$.

But every entire function is of bounded index on any compact set [2b, Theorem 16, p. 305]. Therefore, there is an integer $N_{2} \geq 0$ such that

$$
\frac{\left|f_{k}{ }^{(j)}(z)\right|}{j !} \leq \max _{0 \leq i \leq N_{2}} \frac{\left|f_{k}{ }^{(i)}(z)\right|}{i !} \text { for all integers } j \geq 0
$$

$k=1,2, \ldots, n$, and all $z$ such that $|z| \leq T$. Choosing $N \geq \max \left(N_{1}, N_{2}\right)$, we have (1.1) for all integers $j \geq$ 0 and all $z \in C$.

Theorem. 4.1 does not tell us whether or not the components of $F$ are of bounded index. We conjecture that they are of bounded index; however, at present, we can prove this only for the case $n=2$.

Theorem 4.2: If $F=\left[f_{1} f_{2}\right]$ is an entire solution of $F^{\prime}=A F+Q$ where $A=\left[r_{i j}\right]$ is a matrix whose entries are rational functions which are bounded at infinity and $Q$ is a column vector whose entries are rational functions which are bounded at infinity, then $f_{1}$ and $f_{2}$ are of bounded index.

Proof: If $r_{12} \equiv 0$, then $f_{1}$ satisfies $f_{1}{ }^{\prime}=r_{11} f_{1}+q_{1}$ and by [6], $f_{1}$ is of bounded index.

If $r_{12} \neq \equiv 0$, then $f_{1}$ satisfies

$$
f_{1}^{(2)}=\left(r_{11}+\frac{r_{12}^{\prime}}{r_{12}}+r_{22}\right) f_{1}^{\prime}+\left(r_{11}^{\prime}+r_{12} r_{21}-r_{11} \frac{r_{12}^{\prime}}{r_{12}}-r_{11} r_{22}\right) f_{1}-\left(\frac{r_{12}^{\prime}}{r_{12}}+r_{22}\right) q_{1}+r_{12} q_{2}+q_{1}^{\prime}
$$

and again by [6], $f_{1}$ is of bounded index.

Similarly, $f_{2}$ is of bounded index. 


\section{References}

[1] Gross, F., Entire functions of bounded index, Proc. Amer. Math. Soc. 18 (1967), pp. 974-980.

[2a] Lepson, B., Differential equations of infinite order, hyperdirichlet series and entire functions of bounded index, Lecture notes, 1966, Summer Institute on Entire functions, Univ. of California, La Jolla.

[2b] Lepson, B., Differential equations of infinite order, hyperdirichlet series and entire functions of bounded index, Proc. Sympos. Pure Math. Vol. XI, Amer. Math. Soc., Providence, R. I. (1968), pp. 298-307.

[3] Moulton, F. R., Differential Equations (Macmillan, New York, 1930).

[4] Plemelj, J., Problems in the sense of Riemann and Klein (Interscience, New York, 1964).

[5] Shah, S. M., Entire functions of bounded index, Proc. Amer. Math. Soc. 19 (1968), pp. 1017-1022.

[6] Shah, S. M., Entire functions satisfying a linear differential equation, J. Math. Mech. 18 (1968/69), pp. 131-136.

[7] Shah, S. M., On entire functions of bounded index whose derivatives are of unbounded index, J. London Math. Soc. (2) 4 (1971), pp. 127-139. 